Research Article

\title{
Estimating the Intensity of Hurricanes from Historical Radar Data Using the Hyperbolic-logarithmic Approximation of Spiral Rainbands
}

\author{
Boris S. Yurchak*
}

Environmental Protection Agency, Washington, DC 20460, USA; E-Mail: Yurchak.Boris@epa.gov

* Correspondence: Boris S. Yurchak; E-Mail: Yurchak.Boris@epa.gov

Academic Editor: Alfredo Moreira Caseiro Rocha

Special Issue: Remote Sensing on Climate Change

Adv Environ Eng Res

2020, volume 1 , issue 4

doi:10.21926/aeer.2004002
Received: July 28, 2020

Accepted: October 29, 2020

Published: November 10, 2020

\begin{abstract}
To increase the amount of information on the intensities of tropical cyclones (TC) used in climate research, the possibility of additional estimates of the intensity of a TC by exploring historical data of conventional (non-Doppler) airborne and coastal radars is considered. Based on the hyperbolic-logarithmic spiral (HLS) model of the streamline in the TC, an assessment of the maximum wind speed in hurricanes Cleo (1958), Carolina (1975) and Alicia (1983) was made. Literature sources containing radar signatures of spiral cloud-rain bands (SCRBs) of these hurricanes and the corresponding results of synchronous aircraft soundings were used. The HLS-approximation of the radar signature of the SCRB consisted of determining the "expected" (mean) spiral of a set of HLSs "fitted" into a pattern of the signature. The maximum wind speed was determined from coefficients of the mean HLS. The estimates obtained were in satisfactory agreement with in situ aircraft measurements. The considered examples manifest the possibility of applying the HLS-approximation to determine the intensity of hurricanes by using the historical radar data with satisfactory accuracy.
\end{abstract}

\section{Keywords}

Hyperbolic-logarithmic spiral; tropical cyclone; hurricane; rainbands; radar

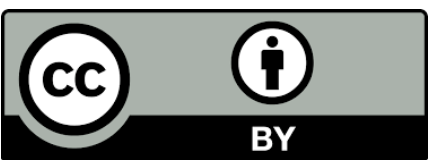

(C) 2020 by the author. This is an open access article distributed under the conditions of the Creative Commons by Attribution License, which permits unrestricted use, distribution, and reproduction in any medium or format, provided the original work is correctly cited. 


\section{Introduction}

Due to the comparative rarity of tropical cyclones (TC), the studying of their response to climate change, in general, and, in particular, to the component caused by anthropogenic factors, is a difficult task (e.g., [1]). One of the difficulties is obtaining data on the properties of a TC, especially its intensity in the "pre-satellite era" [2]. In this paper, the possibility of applying the hyperbolic-logarithmic spiral (HLS) approximation to historical radar data acquired by conventional (non-Doppler) airborne and coastal radars at the end of the "pre-satellite era" and at the initial of the "radar era" (between 1950-1985) is considered. The main method obtaining information about the intensity of a TC over the sea within this period was the in-situ measurements during the aircraft sounding. It seems that non-Doppler radar data were mainly auxiliary information that one can try to use to supplement in situ measurements. The HLS approximation (a brief description of it is provided below) allows one to estimate the intensity of the TC only by the radar reflectivity pattern of the cloud-rain field of the cyclone, which includes spiral cloud-rain spiral bands (SCRBs). These data can be used also in conjunction with the Doppler data to improve the trustworthiness of the maximum wind estimates. In brief, the HLS-approximation is based on the following. In studies of the characteristics of SCRBs detected on radar and satellite imageries of TCs, it was shown that the orientation of the SCRB occurs mainly along the streamlines in the hurricane (e.g., [3-6]). Using the known distribution of forces in a cyclone and representing the cyclone as the Rankine vortex, the analytical expression for the streamline configuration in the form of the HLS was obtained in [7]. (This derivation is also given in the review article [8]). By providing the several examples of satellite and radar imageries of TCs in [7-10], it was shown the presence of spiral signatures of the SCRB, which were satisfactory approximated by the HLS. Since HLS parameters are functions of the Rankine vortex parameters, including the maximum wind speed, the intensity of several cyclones was accordingly estimated in the cited studies using the calculated HLS parameters. These estimates were turned out to be close to both the results of aircraft measurements and the Best Track data including the estimates of the most common empirical method of Dvorak [11]. To test the similar possibility of obtaining the intensity estimates based on historical radar data including its validation, it is necessary to have an image of a spiral complex and data of direct aircraft (in situ) measurements of tropical cyclone characteristics coinciding in time and location with radar measurement data. Based on the literature, three studies of tropical cyclones accompanied by radar measurements of horizontal distribution of reflectivity and in situ measurements of the tangential wind profile have been reported. One report studied hurricane Cleo (1958) [12]. In this study, the composite radar structure of the hurricane and horizontal wind profiles are presented. Another reported experiment was the radaraircraft sounding of hurricane Caroline (1975) on August 8 1975, before it made landfall on the coast of Texas $[13,14]$. A similar experiment was the radar-aircraft sounding of hurricane Alicia (1983) on August 181983 [15] where the wind was measured also by the Doppler radar. The subject of this work is a demonstration of the possibility of using the aforementioned literature data as an example of estimating the intensity of TC using data from conventional radars. 


\section{The Principle of HLS-assessment of the Physical Characteristics of a Tropical Cyclone}

The determination of the TC intensity based on the representation of the streamline in the form of the hyperbolic-logarithmic spiral (HLS) is based on the hyperbolic distribution of the tangential velocity in the outer part of the Rankine vortex

$$
V(r)=V_{m}\left(r_{m} \frac{1}{r}\right)^{n}, \quad r_{m} \leq r<\infty
$$

where $r$ is the polar radius centered in the center of the cyclone, $r_{\mathrm{m}}$ is the radius of the maximum wind relative to which the vortex region is divided into internal and external parts, $V_{\mathrm{m}}$ is the maximum wind speed, $V(r)$ is the wind speed at a distance $r$ from the center of the cyclone, $n$ is the hyperbolic index. Within the outer part of the vortex $n=1$ for an ideal (theoretical) Rankine vortex, and the wind speed varies according to the hyperbolic law. Real TCs differ from the theoretical model due to various reasons and, as experimental data show, the real hyperbolic index varies over a range $n=0.3-0.8$, including its depending on the height $[16,17]$. A streamline for the external section of the Rankine vortex is described by the HLS in polar coordinates originated in the centre of a TC $[7,8]$ :

$$
\phi=A\left(\frac{1}{y^{n+1}}-1\right)-B \ln y \equiv A\left\{e^{-(n+1) \ln y}-1\right\}-B \ln y,
$$

where $\varphi$ is the polar angle and $y=r / r_{0}$ is the polar radius normalized to $r_{0}$ that is the conditional range of the beginning of a streamline which coincides with the range of the accepted reference point of the polar angle of the spiral (a point within a spiral signature). Coefficients A and B in (2) are determined by the following relationships:

$$
A=\frac{r_{m}^{n}}{k(n+1) r_{0}^{n+1}} V_{m}
$$

and

$$
B=\frac{f}{k}
$$

where $k$ is the friction factor, $f$ is the Coriolis parameter. Thus, the parameter $A$, which determines the difference between the HLS and the logarithmic spiral, is proportional to the maximum wind speed. The HLS reflects an increase in cyclone intensity as a decrease of the steepness of the spiral twist as it approaches the center of a TC. At this, the configuration of the spiral adjacent to the central cloudy field of the TC is increasingly degenerating into a circular arc. Expression (2) shows that the HLS is close to the logarithmic spiral, but only on the periphery of the cyclone, where the normalized polar radius is slightly less than 1 . As one approaches to the center of the cyclone, the spiral begins to "round off" (with constant $k$ and $n$ ). The maximum wind speed is higher, the earlier (at a greater distance from the center) this happens. Thus, as follows from the definitions of the HLS coefficients (3) and (4), the maximum wind speed and the friction coefficient can be determined for given $n, f, r_{\mathrm{m}}$, and $r_{0}$ as a function of approximate estimates of the coefficients $\hat{A}$ and $\hat{B}$. 


\section{Assimilation Approximation of a Spiral Band by a Hyperbolic-logarithmic Spiral (HLS- estimation)}

The following is a brief description of the previously developed procedure for the assimilation approximation of a spiral band of a TC with a finite width $[8,10]$. The raw data for the application of the HLS method is a radar image of a TC from weather radar's Plan Position Indicator (PPI). In the current study, the images provided in the articles cited in Introduction section were used. Assimilation approximation is based on two main points. First: a spiral band of finite width, visible on the radar image of a TC, is a display of the result of the involvement of cloud particles in the streamline contribution range. Second: cloudy-rain particles trace a streamline and their real trajectories are replaced (assimilated) by hyperbolic-logarithmic spirals (HLSs). In this case, the HLSs which have reasonable (real) physical parameters are considered to be "equally possible". This circumstance does not allow the use of classical regression analysis, and therefore the approximation consists in determining the all HLSs which "fit" into the geometric boundaries of the spiral band and calculating the "mean HLS" from their set. This determination is conducted by computer drawing of trial HLSs from the initial end of a SCRB within the selected spiral band borders (details are provided below). Fit spirals are defined as "signature" HLSs. A signature HLS has coefficients $A(3)$ and $B(4)$ determined by different combinations of $V_{\mathrm{m}}, k$ and $n$. This triple of parameters is hereinafter referred to as the term "physical characteristics". For the most reliable estimates of the triple $V_{\mathrm{m}}, k$ and $n$, their values of the corresponding mean HLS are taken. Uncertainties in estimating mean values are determined by the root-mean-square "scatter" of parameters of signature HLSs. Since the signature has a finite width, the point of generation of the trial (test) HLSs is selected within the space interval connecting the near and far boundaries of the signature along the corresponding beam of the polar angular coordinate system. This segment is called the signature connector with the corresponding number. The tail (initial) parts of the SCRBs signatures are sometimes not suitable for use in the HLS approximation procedure for various reasons. Therefore, the connector from which the generation of trial HLSs is established is determined experimentally, starting from the center furthest from the center. The suitability of the connector for approximation is established by the emission of a large number of trial HLSs from it (as a rule, more than 1000). The starting point for the emission of trial HLSs is usually selected in the middle of the connector. To limit non-physical HLSs, i.e. signature spirals corresponding to wind speeds close to zero or, conversely, unlikely high speeds, the procedure of consecutive approximations is applied. The procedure is based on the assumption that the wind speed in the band is distributed according to a single-mode law close to the normal (Gauss) law. Therefore, by varying the intervals of variation of the three physical parameters, one obtains the resulting histogram of the distribution of the maximum wind speed of signature HLS close to this or, in any case, a single-mode distribution with a histogram value close to zero at the boundaries of the selected speed range. As practice has shown, the most conservative parameter is the range of the hyperbolic index that is almost always chosen in the range of 0.3-0.8. With a selected range of speeds, the distribution histogram is reduced to a single-mode view, mainly by varying the range of variation of the friction coefficient. The average values of the parameters $\left\langle V_{m}\right\rangle,\langle n\rangle$ and $<k>$ are considered to be estimates of the physical characteristics of the analysed signature, to which the mean HLS corresponds. Additionally, the distribution mode of the maximum velocity $V_{\mathrm{m} \_m d l}$ is estimated. In the case of a symmetrical wind distribution across the band, the modal and 
mean values of the maximum speed are close in magnitude. Due to their large number, the signature HLSs (trial HLSs which are fitted into a spiral band) are displayed over the image of a SCRB as a spiral band of a regular shape in the form like a "comet tail". The mean HLS is shown as a single spiral similar (but not necessarily equal) to the center line of the spiral band of the signature HLS family. Detailed flowchart of the data processing is provided in Supplementary material 1. The above procedure of such a special approximation is referred to as the "HLSestimation", and the results of data processing using this procedure are referred to as the "HLSestimates".

\section{HLS-estimation of TC Intensity Utilizing Historical Literature Radar Data}

\subsection{Hurricane Cleo (1958)}

\subsubsection{Radar-aircraft Data of Hurricane Cleo}

Hurricane Cleo was studied by three aircraft-laboratories on August 181958 between 1600 GMT and $2200 \mathrm{GMT}$. At that time, the hurricane was located approximately $400 \mathrm{n}$. miles east northeast of Bermuda in the Atlantic Ocean. A description of the experiment is reported in [12]. In particular, with the help of a radar mounted on an airplane that performed sounding at maximum height, a composite radar imagery of the horizontal structure of a hurricane in the layer from the ocean surface to a maximum sounding height of 35000 feet $(240 \mathrm{mb})$ was obtained, Figure 1 (Figure 3 of the original article [12]).
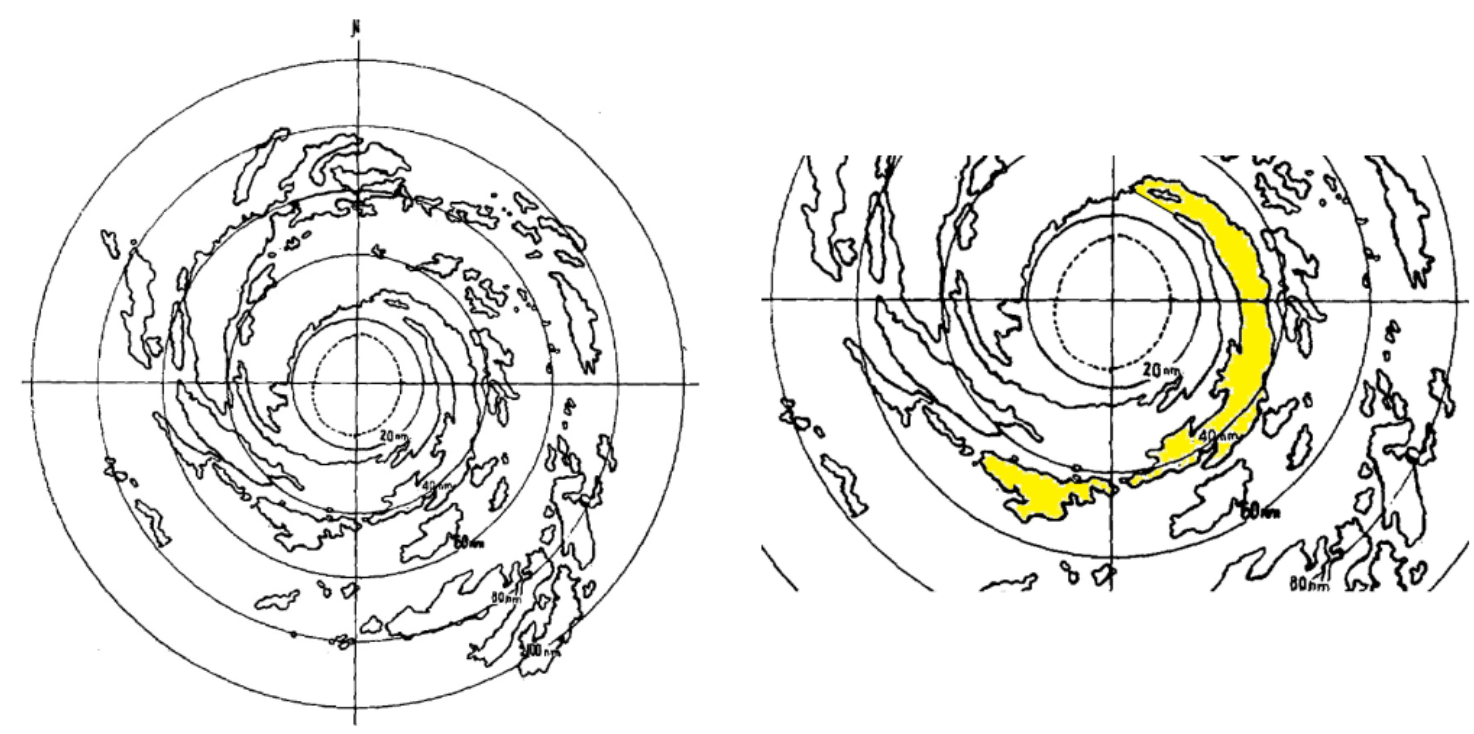

Figure 1 Left panel: Composite radar imagery of Hurricane Cleo, August 181958. (Figure is from [12], La Seur \& Hawkins. 1963.). CAmerican Meteorological Society. Used with permission. Right panel: Fragment of the composite imagery with highlighted principal rainband of the spiral complex.

As follows from the above Figure 1, the pronounced spiral shape has the main SCRB, which was used for the HLS approximation. In the cited study, data on physical parameters, in particular, the profile of horizontal wind speed, were obtained for three levels of 7000, 17000 and 35000 feet (800, 560 and $240 \mathrm{mb}$, respectively) in the direction from west to northeast through the center of 
the hurricane (Figure 2 of the original article [12]). The aircraft route was \pm 100 miles from the center of the hurricane. Wind profiles according to a one-time flight at all heights turned out to be asymmetric. Since the principal band is located at approximately at level $800 \mathrm{mb}$ [6] and it was located mainly in the eastern sector (Figure 1), then the nearest accessible northeastern part of the horizontal wind profile was selected at a level of $800 \mathrm{mb}$ from Figure 2 of [12]. At $800 \mathrm{mb}$ level, the speed and radius of the maximum wind zone of the profile branch from the center of the hurricane to the northeast were 22.7 miles $(42 \mathrm{~km})$ and $90 \mathrm{kts}\left(46.3 \mathrm{~m} \mathrm{~s}^{-1}\right)$, respectively. The least squares approximation of this section of the profile at a distance of 42 to $72 \mathrm{~km}$ gave an estimate of the hyperbolic index $n=0.42 \pm 0.04$.

\subsubsection{HLS-estimates of Maximum Wind of Hurricane Cleo}

The HLS-approximation was applied to the principal band according to the method described above. Signature spirals for the initial signature connector (No. 0) are shown in Figure 2. The total number of signature spirals was 10567.

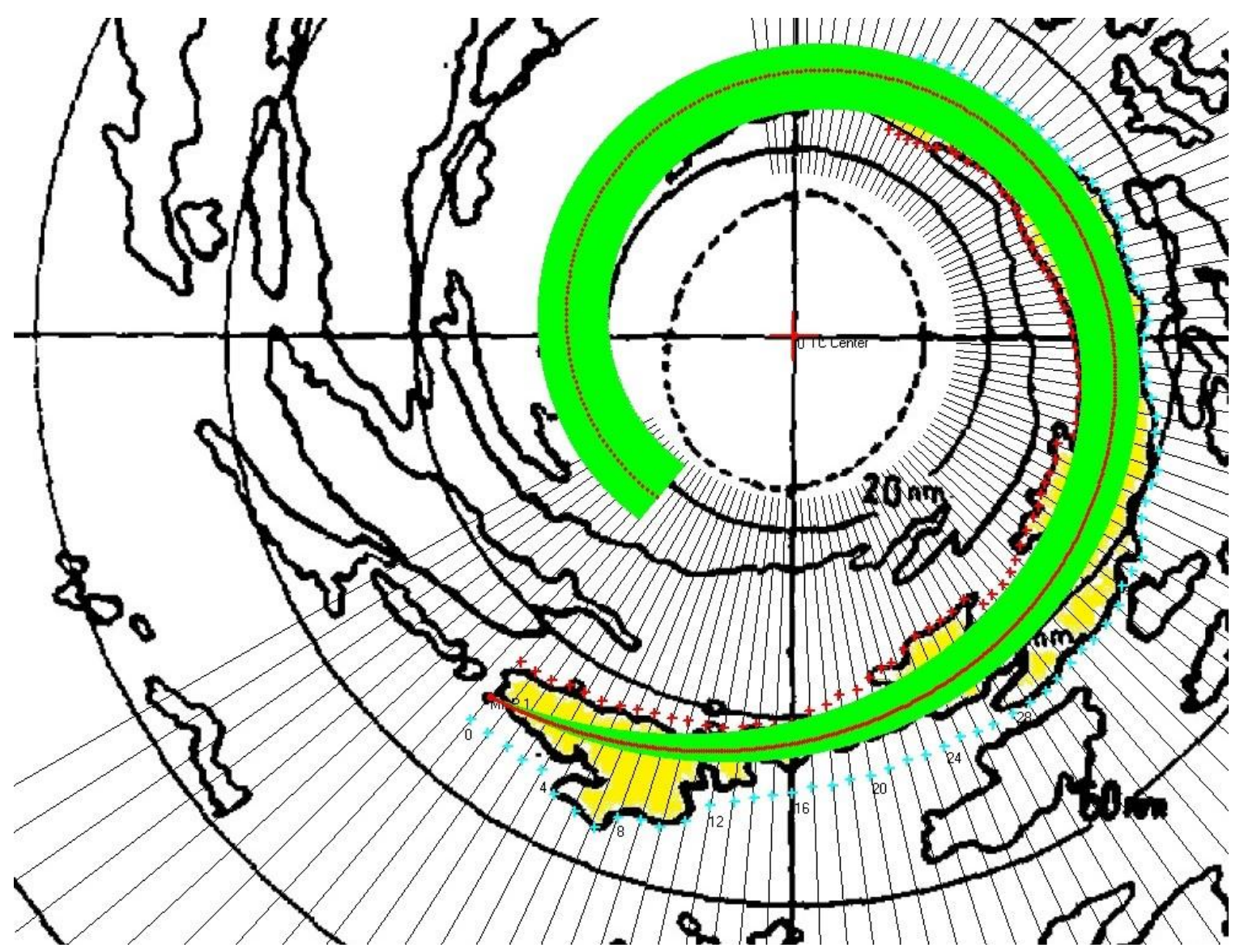

Figure 2 Illustration of HLS-approximation of principal rainband of Hurricane Cleo by means of allocation of signatural HLSs (regular spiral band expanding towards the centre of the cyclone) originated from the initial signatural connector (No. 0). Mean spiral is shown in red. Remaining explanations are in the text.

Average maximum wind speed data, estimates of the hyperbolic index and the friction factor are shown in Table 1 for the principal rainband. 
Table 1 Comparative data of direct measurements (in situ) of physical characteristics of Hurricane Cleo during aircraft sounding and results of the HLS-approximation of the principal rainband.

\begin{tabular}{ccccc}
\hline Parameter & \multicolumn{3}{c}{ HLS-estimate } & In situ \\
\hline$<V_{\mathrm{m}}>, \mathrm{m} \mathrm{s}^{-1}$ & $(\mathrm{kts})$ & $47.6 \pm 1.0$ & $(92.5 \pm 1.9)$ & $46.3(90.0)$ \\
$V_{\mathrm{m} \_\mathrm{mdl}, \mathrm{m} \mathrm{s}^{-1}}$ & $(\mathrm{kts})$ & $42.2 \pm 2.0$ & $(82.0 \pm 3.9)$ & N/A* \\
$<k>10^{5}, \mathrm{~s}^{-1}$ & & \multicolumn{2}{c}{$9.4 \pm 0.2$} & $0.42 \pm 0.04$ \\
$<n>$ & & \multicolumn{2}{c}{$0.57 \pm 0.01$} & \\
\hline
\end{tabular}

${ }^{*)} \mathrm{N} / \mathrm{A}$ - Not Applicable

As follows from table 1 , the obtained results indicate a satisfactory coincidence of the HLSestimates with in-situ data on the maximum wind speed. Their discrepancy is $+1.3 \mathrm{~m} \mathrm{~s}^{-1}$ (2.5 kts) for the average velocity (3\%) and $-4.1 \mathrm{~m} \mathrm{~s}^{-1}$ ( $8 \mathrm{kts}$ ) ( 9\%) for the modal velocity value. The indicated discrepancy between the velocities, and between the hyperbolic indexes by 0.15 ( 36\%) could be due to the wind sensing route was to the northeast, which is not quite adequate to the location of the principal band. It would be preferable to have an orientation of the route from the centre to the east or southeast, corresponding to the region of formation of the principal rainband. The mean HLS shown in red in Figure 2 has coefficients $\langle A\rangle=6.16$ and $\langle B\rangle=0.85$.

\subsection{Hurricane Caroline (1975)}

\subsubsection{Radar-aircraft Data of Hurricane Caroline}

At the time the aircraft crossed the centre of Hurricane Caroline at 1830 GMT on 30 August 1975, the hurricane's eye was approximately $260 \mathrm{~km}$ from the meteorological radar site in Brownsville, Texas, making it possible to obtain a radar horizontal section of the cyclone with spiral rainbands adjacent to the eye, Figure 3. Coordinates of the radar were: $25.916^{\circ} \mathrm{N}, 97.419^{\circ}$ $W$. Coordinates of the centre of the hurricane at the time of the aircraft passing through its centre were: $23.87^{\circ} \mathrm{N}, 96.33^{\circ} \mathrm{W}$. Simultaneously, the wind profile was measured using airborne sounding at an altitude of $450 \pm 100 \mathrm{~m}$ through the geometric centre of the hurricane. Airborne sounding was carried out from the West (W) to the East (E) along a straight track through the geometric centre of the hurricane. The wind profile was asymmetric according to the data of the one-time flight. The western part had $V_{\mathrm{m}}=31 \mathrm{~m} \mathrm{~s}^{-1}(60.3 \mathrm{kts}), R_{\mathrm{m}}=37 \mathrm{~km}$; and the eastern part had $V_{\mathrm{m}}=46$ $\mathrm{m} \mathrm{s}^{-1}(89.4 \mathrm{kts}), R_{\mathrm{m}}=27.8 \mathrm{~km}$ [14]. Using the above-mentioned results of the tangential wind profile measurements along a distance of approximately $40 \mathrm{~km}$ (Figure 6 in [13]), the hyperbolic index (n) was determined to be approximately 0.44 for the western sector and 0.52 for the eastern sector. The wind speed data from measurements, which carried out during several flights in the direction to the right and perpendicular to the trajectory of the movement of the hurricanes centre (along a flight track of about $170 \mathrm{~km}$ ) as it approached the coast, are provided in Figure 12 in [13]. It follows from these data that $V_{\mathrm{m}} \approx 47.4 \mathrm{~m} \mathrm{~s}^{-1}(92.1 \mathrm{kts}), R_{\mathrm{m}}=29.1 \mathrm{~km}$ and $n=0.53$. These results are similar to the above-mentioned data obtained for a single flight in the eastern sector. Figure 3 shows a part of the radar image of the hurricane reproduced from Figure 5 in [14], which refers to its core and two rainbands. The two regular spirals superimposed on the principal and 
secondary bands in the figure belong to the original drawing and represent (according to the cited article) logarithmic spirals with the crossing angle of $18^{\circ}$.

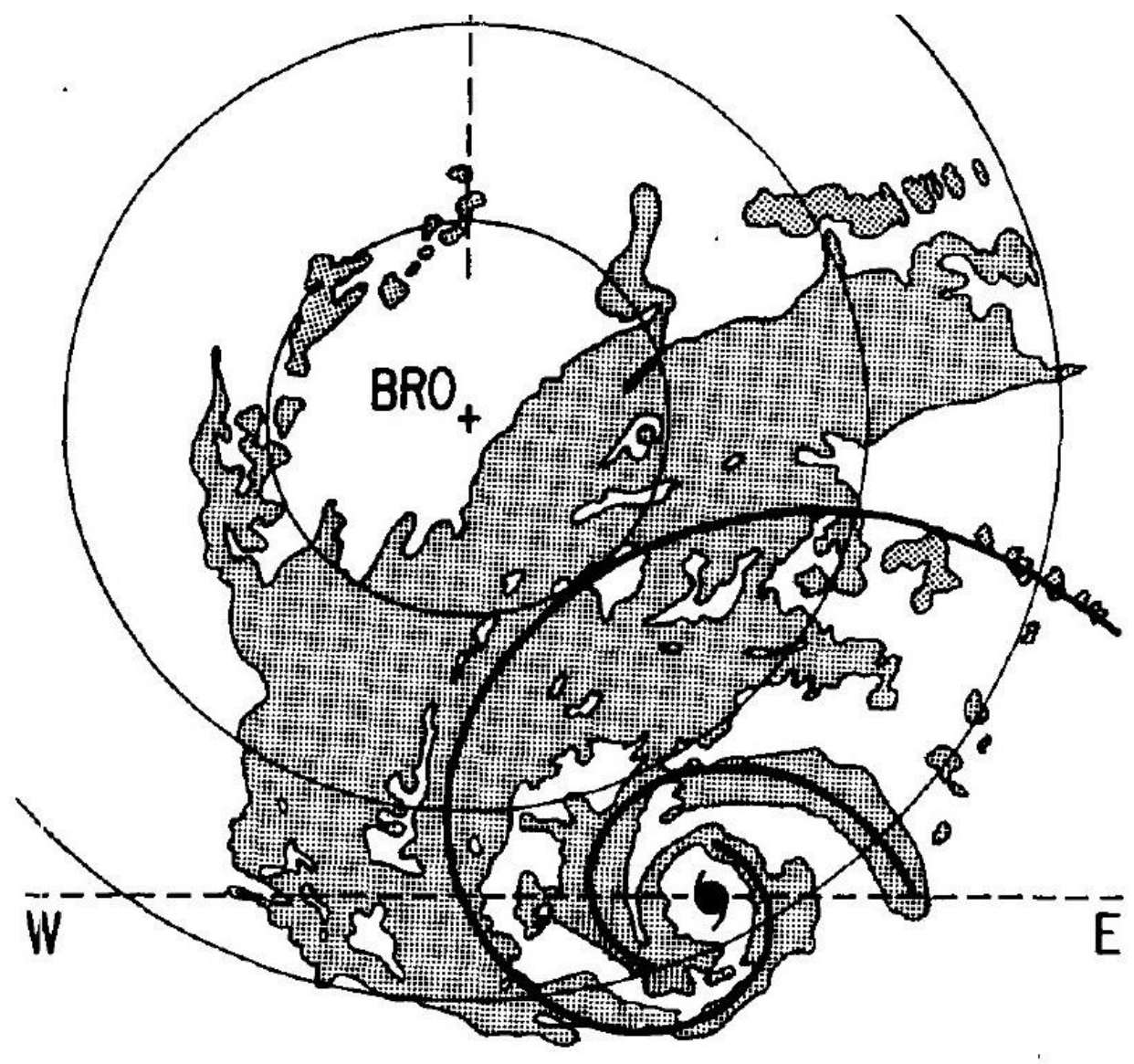

Figure 3 Radar imagery of Hurricane Carolina at 1830 GMT 30 August 1975 from radar installed in Brownsville (BRO), Texas. The range marks are depicted over 50 n.m. (92.6 km) (Figure is from [14], Willoughby. 1978), (CAmerican Meteorological Society. Used with permission. Remaining explanations are in the text.

\subsubsection{HLS-estimates of the Maximum Wind of Hurricane Caroline}

Analysis of Figure 3 shows that the most pronounced SCRB, suitable for HLS approximation, is the secondary band located in the eastern sector. Therefore, only this band was selected for analysis. Figure 4 shows the result of its filling with signature HLSs ( 5575 spirals). Due to the partial overlapping of signature spirals, the family of signature spirals is depicted in the figure as a light green spiral band starting in the middle of signature connector No. 0 and then diverging as it approaches the center of the cyclone. The red spiral inside this signature band is the mean signature HLS. The Rankine vortex model, on the basis of which the HLS was derived, assumes only a symmetric distribution of wind speed and, of course, only one value of the radius of the maximum wind zone. Because of that, the average value of the maximum wind radius based on the data of a one-time flight was found to be approximately $32 \mathrm{~km}$. 


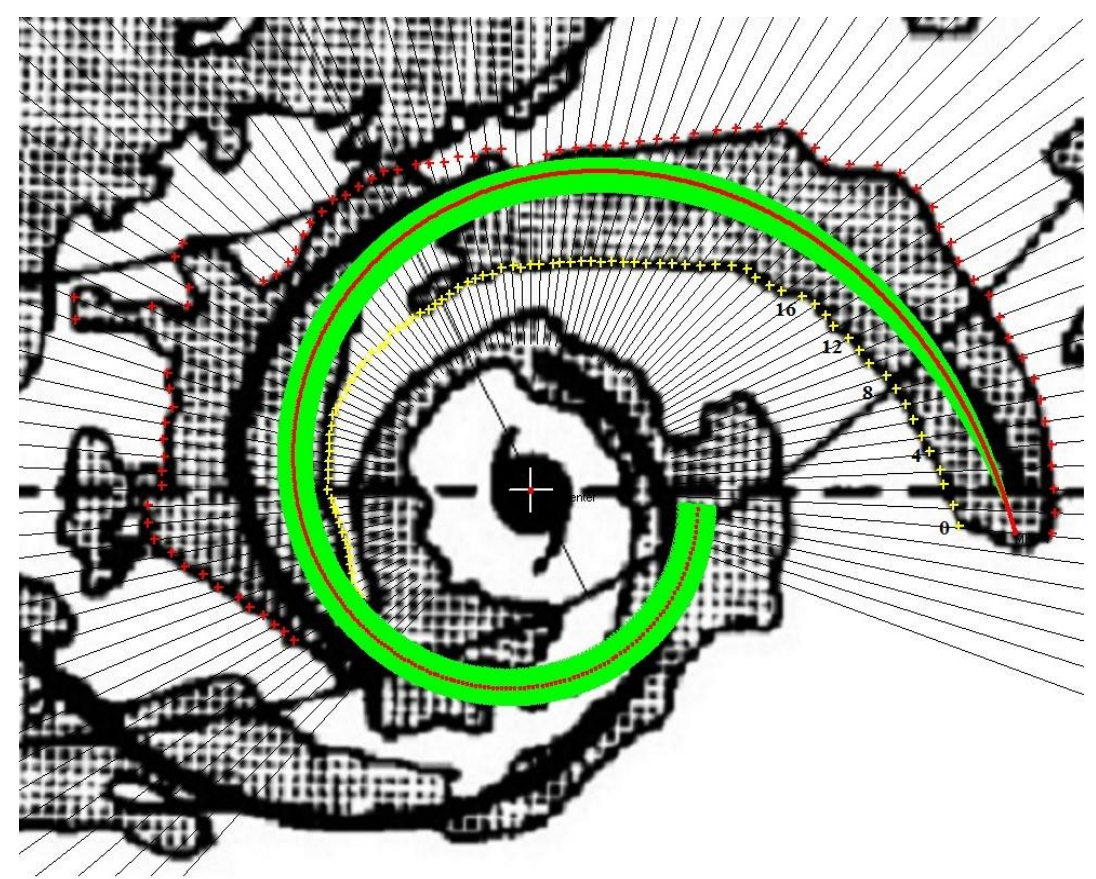

Figure 4 Illustration of the HLS approximation of the secondary spiral band of the eastern sector of the radar imagery of Hurricane Carolina. A family of signature HLSs is shown as a light green spiral band expanding toward the center of the cyclone. The mean signature spiral is indicated in red. The remaining explanations are in the text.

The HLS-estimates are shown in Table 2. In the same table, for comparison, the data of direct measurements (in situ) during airborne sounding are shown.

Table 2 Comparative data of direct measurements (in situ) of physical characteristics of Hurricane Carolina resulted from aircraft sounding and results of the HLSapproximation.

\begin{tabular}{|c|c|c|c|c|c|c|}
\hline \multirow{3}{*}{ Sector } & \multicolumn{3}{|c|}{$V_{\mathrm{m}}, \mathrm{m} \mathrm{s}^{-1}$ (kts) } & \multicolumn{2}{|c|}{$n$} & \multirow{3}{*}{$\frac{k \cdot 10^{5}, s^{-1}}{H L S}$} \\
\hline & \multirow{2}{*}{ In-situ } & \multicolumn{2}{|c|}{ HLS } & \multirow{2}{*}{ In-situ*** } & \multirow{2}{*}{ HLS } & \\
\hline & & Mean & Modal & & & \\
\hline East & $46 \quad(89.4)^{*}$ & $47.6 \pm 0.4$ & $49.5 \pm 5.5$ & $0.52 \pm 0.03$ & & \\
\hline Traverse & $47.4(92.1)^{* *}$ & $(92.5 \pm 0.8)$ & $(96.2 \pm 10.7)$ & $0.53 \pm 0.02$ & $0.54 \pm 0.01$ & $11.4 \pm 0.1$ \\
\hline
\end{tabular}

*) From [14], p. 845

**) From [13], Figure 12, p. 1304

${ }^{* * *}$ ) Estimates based on graphical data given in Figure 6 and Figure 12 from [13]

The mean signature HLS has coefficients $\langle A\rangle=3.95 n\langle B\rangle=0.52$. As follows from table 2 , the obtained results indicate a satisfactory coincidence of the HLS estimates and in-situ data on the maximum wind speed and the hyperbolic index. 


\subsection{Hurricane Alicia (1983)}

\subsubsection{Radar-aircraft Data of Hurricane Alicia}

Hurricane Alicia was probed between 0108 and 0200 UTC on August 18 1983, when it was in the Gulf of Mexico (28.5 $\mathrm{N}$ and $94.8^{\circ} \mathrm{W}$; about $90 \mathrm{~km}$ south of Galveston, Texas). Maximum wind speeds recorded by the aircraft Doppler radar at a flight altitude of $1500 \mathrm{~m}$ were $50-53 \mathrm{~m} \mathrm{~s}^{-1}$ (97.2$103.0 \mathrm{kts})$, with the minimum central pressure of $967 \mathrm{mb}$ [15]. The maximum wind radius from these measurements was approximately $18 \mathrm{~km}$. A radar image of Hurricane Alicia from PPI of meteorological radar WSR-57 at Galveston is shown in Figure 5.

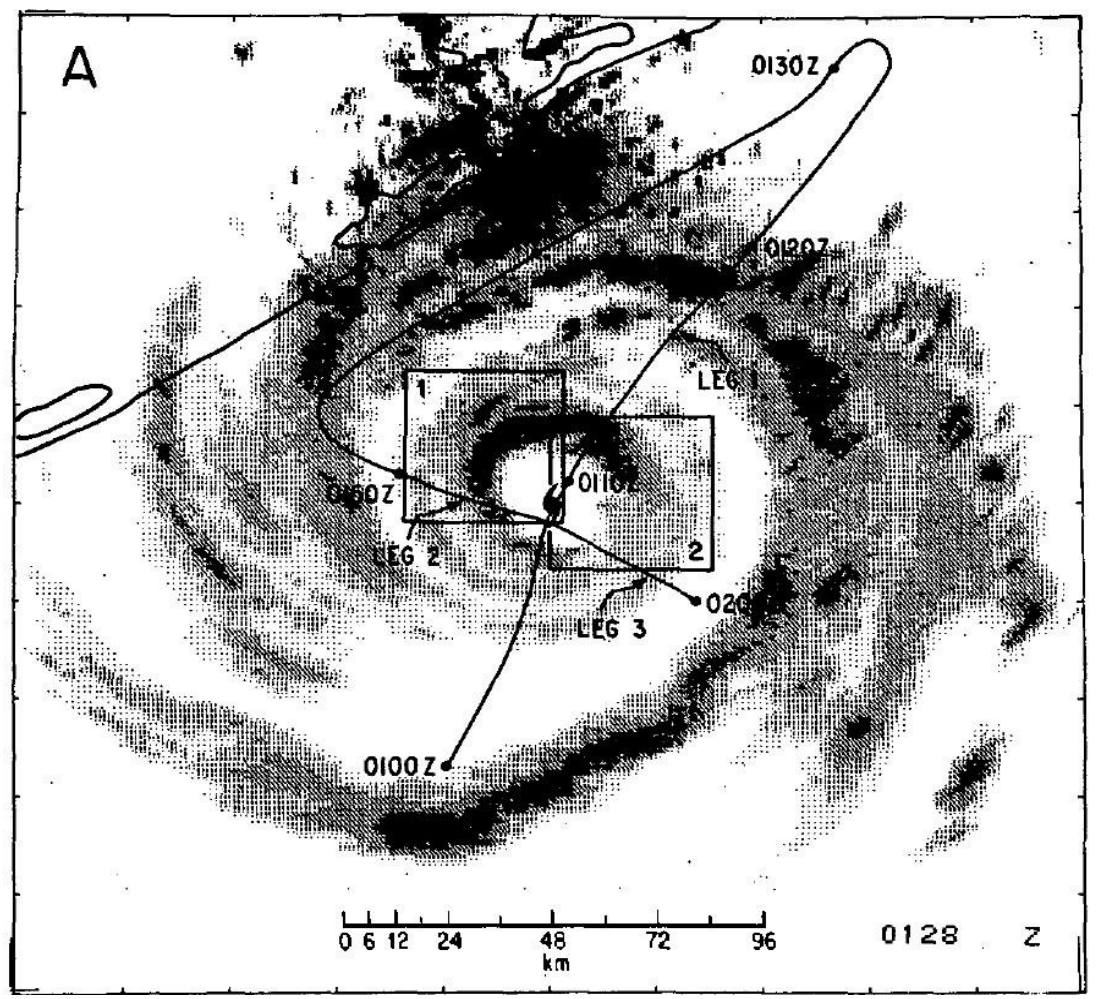

Figure 5 Radar imagery of Hurricane Alicia (1983) obtained at 0128 UTC (Figure is from [15], Marks \& Houze. 1987). (C) American Meteorological Society. Used with permission. Remaining explanations are in the text.

The stationary spiral complex [4] of Hurricane Alicia consists of a secondary rainband extending in the direction from the south to the northeast, and the principal rainband starting in the eastern sector of the image. The hyperbolic index was not determined, due to the short distance of the tangential wind profile measurement in the external part of the vortex $(\sim 25 \mathrm{~km})$.

\subsubsection{HLS-estimates of Maximum Wind in Hurricane Alicia}

The HLS-approximation was applied to a fragment of the principal band and to a more extended secondary band. The signature spirals are shown in Figure 6. 

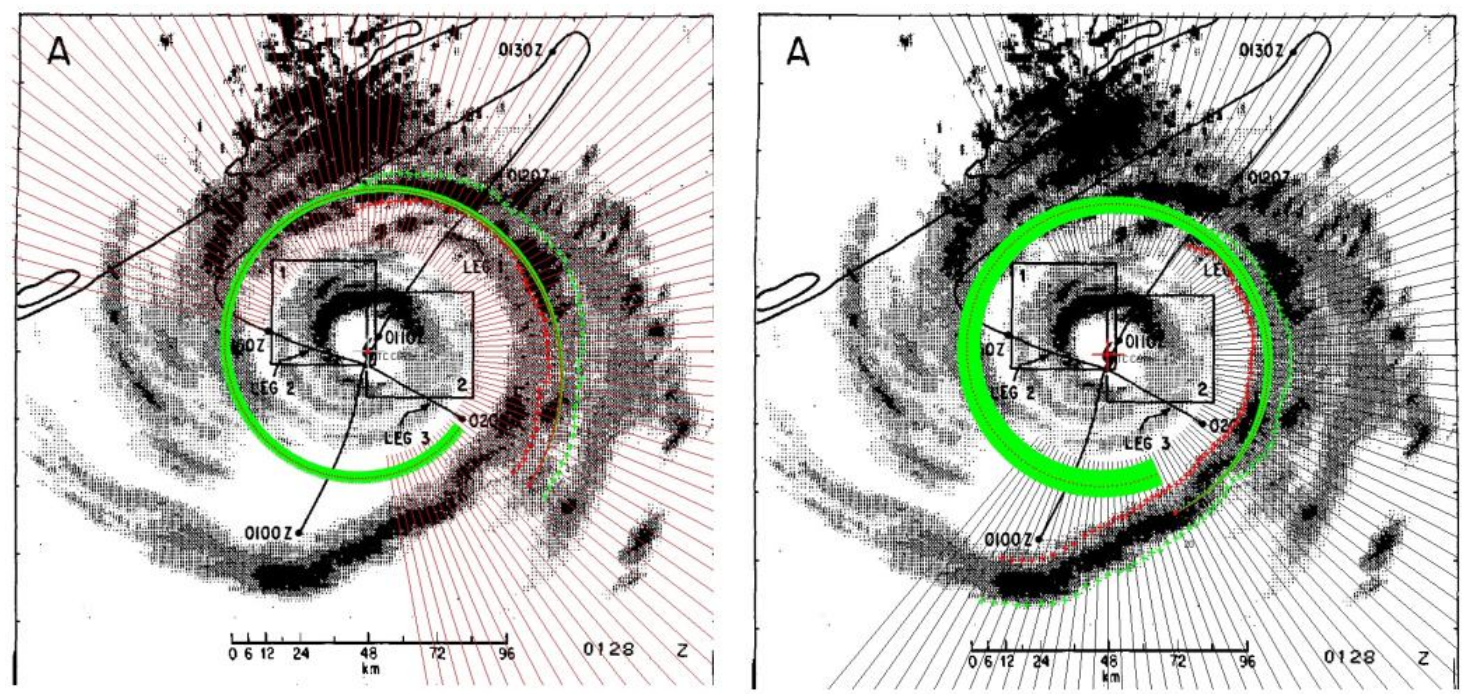

Figure 6 Illustration of the HLS-approximation of spiral rainbands of Hurricane Alicia by means of allocation of signature HLSs. The family of signature HLS is shown as a light green spiral band expanding toward the center of the cyclone. Left panel: HLSapproximation of the principal band from initial signature connector (No. 0); Right panel: HLS-approximation of the secondary band from signature connector No. 20. The mean signature spiral of the principal and secondary bands is marked in red. Remaining explanations are in the text.

The staring signature connector for the principal band was No. 0. For the secondary band, the signature HLSs were available starting from signature connector No. 20. The average maximum wind speed data, hyperbolic index and friction factor are shown in Table 3 for both rainbands. The same table shows the parameters of the mean HLS for both bands.

Table 3 Results of assessment of physical characteristics of Hurricane Alicia by means of the HLS-approximation of the principal and secondary rainbands.

\begin{tabular}{|c|c|c|c|}
\hline \multirow{3}{*}{ Parameter } & \multicolumn{3}{|c|}{ HLS-estimates } \\
\hline & \multicolumn{3}{|c|}{ Rainband } \\
\hline & Principal & \multicolumn{2}{|c|}{ Secondary } \\
\hline$<V_{\mathrm{m}}>, \mathrm{m} \mathrm{s}^{-1}(\mathrm{kts})$ & $(99.5 \pm 1.5)$ & $50.8 \pm 0.3$ & $(98.7 \pm 0.6)$ \\
\hline$V_{\mathrm{m} \_\mathrm{mdl}}, \mathrm{m} \mathrm{s}^{-1}(\mathrm{kts})$ & $(90.4 \pm 8.7)$ & $43.0 \pm 1.0$ & $(83.6 \pm 1.9)$ \\
\hline$<k>\cdot 10^{5}, \mathrm{~s}^{-1}$ & $5.44 \pm 0.06$ & \multicolumn{2}{|c|}{$2.95 \pm 0.01$} \\
\hline$<n>$ & $0.52 \pm 0.01$ & \multicolumn{2}{|c|}{$0.52 \pm 0.01$} \\
\hline \multicolumn{4}{|c|}{$\mathrm{V}_{\mathrm{m}}$ in situ $=50-53 \mathrm{~m} \mathrm{~s}^{-1}$ (97.2-103.0 kts) } \\
\hline \multicolumn{4}{|c|}{ Parameters of mean signature HLS } \\
\hline$<A>$ & 12.06 & \multicolumn{2}{|c|}{29.91} \\
\hline$<B>$ & 1.28 & \multicolumn{2}{|c|}{2.36} \\
\hline $\begin{array}{c}\text { Number of } \\
\text { signature HLSs }\end{array}$ & 1331 & \multicolumn{2}{|c|}{16145} \\
\hline
\end{tabular}


As follows from table 3, the results obtained indicate, as in the cases considered in the previous sections, a satisfactory coincidence of the HLS-estimates both between different bands and with Doppler radar data (50-53 $\mathrm{m} \mathrm{s}^{-1}$ (97.2-103.0 kts) ) on the maximum wind speed in this hurricane. An exception is the estimate of the modal value of the velocity of the secondary band that was approximately $7 \mathrm{~m} \mathrm{~s}^{-1}$ (13.6 kts) lower than the average value for the approximation HLS and the data of direct measurements. The revealed difference is apparently due to the asymmetric distribution of the wind in the section of the secondary SCRB. The case considered is an example of the conjunction utilization of Doppler and non-Doppler radar data.

\section{Summary}

The literature data on direct (in situ) measurements of the intensity of Hurricanes Cleo (1958), Carolina (1975) and Alicia (1983) obtained as a result of airborne and radar soundings are compared with the results of hyperbolic-logarithmic approximation of spiral cloud-rain bands (SCRBs) of these TCs. The processing of the radar signatures of the SCRBs consisted of determining the average over the set of hyperbolic-logarithmic spirals (HLSs) that "fit" into the geometric boundaries of the signatures. A comparison of the results of direct aircraft measurements of the intensity of the considered hurricanes as well as with the Doppler radar data (case of Hurricane Alicia) with the results of the HLS-approximation of the corresponding conventional radar data confirmed their satisfactory agreement. The obtained positive result of the analysis of the considered examples indicates the possibility of performing the HLS-estimates of the intensity of hurricanes with satisfactory accuracy from historical and archival data of radar measurements by conventional radars for their use in climate studies relevant to tropical cyclones.

\section{Acknowledgments}

It is the author's pleasure to acknowledge valuable and useful comments by the three anonymous reviewers of the paper.

\section{Author Contributions}

Boris S. Yurchak did all the research work of this study.

\section{Competing Interests}

The author has declared that no competing interests exist.

\section{References}

1. Knutson T, Camargo SJ, Chan JC, Emanuel K, Ho CH, Kossin J, et al. Tropical cyclones and climate change assessment: Part I: Detection and attribution. Bull Amer Meteorol Soc. 2019; 100: 1987-2007.

2. Vecchi GA, Knutson TR. On estimates of historical North Atlantic tropical cyclone activity. J Clim. 2018; 21: 3580-3600.

3. Kurihara Y. On the development of spiral bands in a tropical cyclone. J Atmos Sci. 1976; 33: 940-958. 
4. Willoughby HE, Marks Jr FD, Feinberg RJ. Stationary and moving convective bands in hurricanes. J Atmos Sci. 1984; 41: 3189-3211.

5. Willoughby HE. The dynamics of the tropical cyclone core. Aust Meteor Mag. 1988; 36: 183191.

6. Houze RA. Clouds in tropical cyclones. Mon Weather Rev. 2010; 138: 293-344.

7. Yurchak BS. Description of cloud-rain bands in a tropical cyclone by a hyperbolic-logarithmic spiral. Russ Meteorol Hydrol. 2007; 32: 8-18.

8. Yurchak BS. The use of a spiral band model to estimate tropical cyclone intensity. Current Topics in Tropical Cyclone Research. London: IntechOpen; 2019. Available from: https://www.intechopen.com/online-first/the-use-of-a-spiral-band-model-to-estimatetropical-cyclone-intensity

9. Yurchak BS. Estimation of tropical cyclone intensity from the satellite infrared images of its spiral cloud bands. Russ Meteorol Hydrol. 2018; 43: 581-590.

10. Yurchak BS. An estimate of the hurricane's intensity from radar data using hyperboliclogarithmic approximation. Int J Remote Sens. 2019; 40: 9629-9641.

11. Dvorak VF. Tropical cyclone intensity analysis and forecasting from satellite imagery. Mon Weather Rev. 1975; 103: 420-430.

12. La Seur NE, Hawkins HF. An analysis of Hurricane Cleo (1958) based on data from research reconnaissance aircraft. Mon Weather Rev. 1963; 91: 694-709.

13. Merceret FJ. The turbulent microstructure of Hurricane Caroline (1975). Mon Weather Rev. 1976; 104: 1297-1307.

14. Willoughby HE. A possible mechanism for the formation of hurricane rainbands. J Atmos Sci. 1978; 35: 838-848.

15. Marks Jr FD, Houze RA. Inner core structure of Hurricane Alicia from Doppler radar observations. J Atmos Sci. 1987; 44: 1296-1317.

16. Gray WM, Shea DJ. The hurricane's inner core region. II. Thermal stability and dynamic characteristics. J Atmos Sci. 1973; 30: 1565-1577.

17. Samsury CE, Zipser EJ. Secondary wind maxima in hurricanes: Airflow and relationship to rainbands. Mon Weather Rev. 1995; 123: 3502-3517.

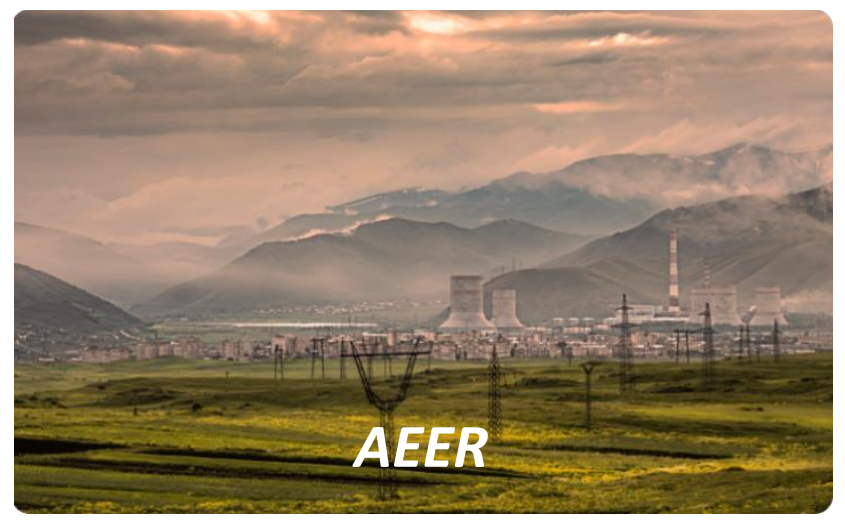

Enjoy $A E E R$ by:

1. Submitting a manuscript

2. Joining in volunteer reviewer bank

3. Joining Editorial Board

4. Guest editing a special issue

For more details, please visit:

http://www.lidsen.com/journals/aeer 